\title{
Anschriftenverzeichnis der Autoren
}

Baumgarten, Claus, PD Dr. med. Hautklinik u. Asthma-Poliklinik Univ.-Klinikum Rudolf Virchow Augustenburger Platz 1, D-13353 Berlin

Bleckmann, Heinrich, Prof. Dr. med.

Augen-Abteilung

Schloßpark-Klinik

Heubnerweg 2, D-14059 Berlin

Blümcke, Sigurd, Prof. Dr. med. Institut für Pathologie

Univ.-Klinikum Rudolf Virchow

Augustenburger Platz 1, D-13353 Berlin

Böcker, Werner, Prof. Dr. med.

Gerhard-Domagk-Institut für Pathologie

Westfälische Wilhelms-Universität

Domagkstr. 17, D-88149 Münster

Breitenecker, Gerhard, Prof. Dr. med.

Institut für Klinische Pathologie

Gynäkopathologie und Zytologie

Allgemeines Krankenhaus

Währinger Gürtel 18-20, A-1090 Wien

Dhom, Georg, Prof. Dr. med.

Am Webersberg 20, D-66424 Homburg/Saar

Dienes, Hans-Peter, Prof. Dr. med.

Pathologisches Institut der Universität

Langenbeckstr. 1, D-55131 Mainz

Falk, Stephan, Dr. med.

Praxis für Pathologie

Neebstr. 6, D-60332 Frankfurt a. M.

Geiler, Gottfried, Prof. Dr. med.

Institut für Pathologie der Universität

Liebigstr. 26, D-04103 Leipzig

Gelderblom, Hans, Prof. Dr. med.

Robert-Koch-Institut

Abt. Virologie

Nordufer 20, D-13353 Berlin

Gottschalk, Joachim, Prof. Dr. med.

Allgemeines Krankenhaus Heidberg

Abteilung für Pathologie

Tangstedter Landstr. 400, D-22417 Hamburg
Grosse, Gernot, Prof. Dr. med.

Pathologisches Institut

Auguste-Viktoria-Krankenhaus

Rubensstr. 125, D-12157 Berlin

Harms, Dieter, Prof. Dr. med.

Pathologisches Institut der Universität Michaelisstr. 11, D-24105 Kiel

Hoder, Dietmar, Dr. rer. nat. Institut für Pathologie Univ.-Klinikum Rudolf Virchow Augustenburger Platz 1, D-13353 Berlin

Holzner, J. Heinrich, em. Prof. Dr. med. Korngasse 47, A-1170 Wien

Jahnke, Volker, Prof. Dr. med. Hals-Nasen-Ohrenklinik u. Poliklinik Univ.-Klinikum Rudolf Virchow Augustenburger Platz 1, D-13353 Berlin

Klöppel, Günther, Prof. Dr. med. Dienst Anatomo-Pathologie Akademisch Ziekenhuis Vrije Universiteit Brussel Laarbeeklaan 101, B-1090 Brüssel

Kunz, Jochen, Prof. Dr. med. Institut für Pathologie Klinikum Ernst von Bergmann Charlottenstr. 72, D-14467 Potsdam

Lobeck, Hartmut, Prof. Dr. med. Institut für Pathologie Univ.-Klinikum Rudolf Virchow Augustenburger Platz 1, D-13353 Berlin

Löning, Thomas, Prof. Dr. med. Abt. für Gynäkologische Histopathologie und Elektronenmikroskopie Martinistr. 52, D-20246 Hamburg

Loy, Volker, Prof. Dr. med. Institut für Pathologie Univ.-Klinikum Benjamin Franklin Hindenburgdamm 30, D-12200 Berlin

Matuschka, Franz-Rainer, Prof. Dr. rer.nat. Institut für Pathologie, Arbeitsbereich Parasitologie Univ.-Klinikum Rudolf Virchow Malteser Str. 74-100, D-12249 Berlin 
Meißner, Kurt, PD Dr. med.

Hautärztliche Praxis

Dermatohistologisches Labor

Tangstedter Landstr. 77, D-22415 Hamburg

Morgenroth, Konrad, Prof. Dr. med.

Pathologisches Institut der Ruhr-Universität

Universitätsstr. 150, D-44780 Bochum-Querenburg

Müller, Klaus-Michael, Prof. Dr. med.

Institut für Pathologie

Berufsgenoss. Krankenanstalten Bergmannsheil

Gilsingstr. 14, D-44789 Bochum

Nizze, Horst, Prof. Dr. med.

Institut für Pathologie

Universität Rostock

Strempelstr. 14, D-18055 Rostock

Otto, H.-F, Prof. Dr. med.

Institut für Pathologie

Universität Heidelberg

Im Neuenheimer Feld 220-221, D-69120 Heidelberg

Pfannkuch, Friedlieb, Prof. Dr. med.

Ciba-Geigy AG

Pharmatoxikologie K-135.1.83

CH-4002 Basel

Radke, Cornelia, Dr. med.

Institut für Pathologie

Univ.-Klinikum Rudolf Virchow

Augustenburger Platz 1, D-13353 Berlin

Remmele, Wolfgang, Prof. Dr. med.

Institut für Pathologie

Kliniken der Landeshauptstadt

Ludwig-Erhard-Straße 100, D-65199 Wiesbaden

Roschlau, G., Prof. Dr. med.

Pathologisches Institut

Städt. Krankenhaus im Friedrichshain

Landsberger Allee 49, D-10249 Berlin

Rumpelt, H.-J., Dr. med.

Pathologisches Institut

Städt. Krankenhaus Heilbronn

Jägerhausstr. 26, D-74074 Heilbronn

Schaefer, Hans-Eckart, Prof. Dr. med.

Pathologisches Institut

Albert-Ludwigs-Universität

Albertstr. 19, D-79002 Freiburg
Schmidt, Dietmar, Prof. Dr. med.

Institut für Pathologie

A2,2

D-68159 Mannheim

Schnoy, Norbert, Prof. Dr. med.

Institut für Pathologie

Univ.-Klinikum Rudolf Virchow

Augustenburger Platz 1, D-13353 Berlin

Scholman, Hans-Joachim, Dr. med.

Institut für Pathologie

Univ.-Klinikum Rudolf Virchow

Augustenburger Platz 1, D-13353 Berlin

Schröder, J. Michael, Prof. Dr. med.

Institut für Neuropathologie

Med. Fakultät der RWTH

Pauwelsstr. 30, D-52057 Aachen

Schulz, Andreas, Prof. Dr. med.

Institut für Pathologie

Klinikum der Justus-Liebig-Universität

Langhansstr. 10, D-35392 Gießen

Stein, Harald, Prof. Dr. med.

Institut für Pathologie

Univ.-Klinikum Benjamin Franklin

Hindenburgdamm 30, D-12200 Berlin

Stutte, H.J., Prof. Dr. med.

Senckenbergisches Zentrum der Pathologie

J.W. Goethe-Universität

Theodor-Stern-Kai 7, D-60596 Frankfurt a.M.

Trautmann, Matthias, PD Dr. med.

Institut für Mikrobiologie und Immunologie

Abt. Bakteriologie

Klinikum der Universität

Steinhövelstr. 9, D-89075 Ulm

Vogel, Martin, Prof. Dr. med.

Institut für Pathologie

Abt. Paidopathologie und Placentologie

Univ.-Klinikum Rudolf Virchow

Augustenburger Platz 1, D-13353 Berlin 